\title{
AN INTERESTING NEW METRIC AND ITS APPLICATIONS TO ALTERNATING SERIES
}

\begin{abstract}
This paper presents one interesting metric discovered while studying convergence of series with alternating signs. In previous papers [D1] and [D2] we presented a study of 'typical' series with alternating signs. Namely, given a sequence of real nonnegative numbers whose sum is infinity we consider all different ways the signs plus or minus could be put in front of each of these numbers. With a given metric we ask what is the 'size' of the set of those choices of + or - for which the resulting series with alternating signs converges. The term 'size' here means either the Baire category or porosity of this set. While metrics studied before as Frèchet, Baire or Euclidean allowed us to get interesting results, they all share one undesirable property - insensitiveness to the change of a 'tail' of a sequence. The D-metric, introduced here, does not have this undesirable property.
\end{abstract}

\section{Introduction}

The problem of relatively convergent series is studied in many monographs and articles. We study the convergence of the series

$$
\sum_{n=1}^{\infty}(-1)^{a_{n}} b_{n}
$$

where $\left(a_{n}\right)_{n \in \mathbb{N}}$ is a sequence of zeros and ones and $\left(b_{n}\right)_{n \in \mathbb{N}}$ is a sequence of nonnegative real numbers. For fixed sequence $\left(b_{n}\right)_{n \in \mathbb{N}}$ we want to consider the

Key Words: the convergence and divergence of series, D-metric, Baire category, porosity Mathematical Reviews subject classification: 28A21, secondary 54E35, 40A05, 54E52 Received by the editors March 31, 2000 
sets

$$
\begin{aligned}
& \mathcal{C}=\left\{\left(a_{n}\right)_{n \in \mathbb{N}} \in\{0,1\}^{\mathbb{N}} ; \text { the series (1) converges }\right\}, \\
& \mathcal{B}=\left\{\left(a_{n}\right)_{n \in \mathbb{N}} \in\{0,1\}^{\mathbb{N}} ; \exists M>0 \forall k \quad\left|\sum_{n=1}^{k}(-1)^{a_{n}} b_{n}\right| \leq M\right\} .
\end{aligned}
$$

The set $\mathcal{C}$ contains all sequences $\left(a_{n}\right)_{n \in \mathbb{N}}$ for which the series above converges, whereas the set $\mathcal{B}$ contains sequences $\left(a_{n}\right)_{n \in \mathbb{N}}$ for which the series (1) has bounded partial sums. Clearly, $\mathcal{C} \subset \mathcal{B}$. The question what these sets are is definitely nontrivial only if the series

$$
\sum_{n=1}^{\infty} b_{n} \text { diverges, }
$$

i.e., has sum infinity, because otherwise the series (1) is convergent for any choice of $\left(a_{n}\right)_{n \in \mathbb{N}}$. Therefore (2) will be our standard assumption in the entire paper.

Since we are working in the space $\{0,1\}^{\mathbb{N}}$ there are several choices of metrics we can consider. In the paper [D1] we defined the function $\varphi:\{0,1\}^{\mathbb{N}} \rightarrow[0,1]$ by

$$
\varphi\left(\left(a_{n}\right)_{n \in \mathbb{N}}\right)=\sum_{n=1}^{\infty} \frac{a_{n}}{2^{n}}, \text { for }\left(a_{n}\right)_{n \in \mathbb{N}} \in\{0,1\}^{\mathbb{N}} .
$$

Then we put $d_{E}(a, b)=|\varphi(a)-\varphi(b)|$. The function $d_{E}$ is a pseudometric on the space $\{0,1\}^{\mathbb{N}}$. By dropping all sequences of the form $\left(a_{1}, a_{2}, . ., a_{n}, 0,1,1,1, \ldots\right)$ we get a set

$$
\mathcal{M}=\{0,1\}^{\mathbb{N}} \backslash\left\{\left(a_{1}, a_{2}, . ., a_{n}, 0,1,1,1, \ldots\right) ; a_{i} \in\{0,1\}, i=1,2, \ldots, n\right\}
$$

on which $d_{E}$ is a metric. Moreover, $\left(\mathcal{M}, d_{E}\right)$ is a complete metric space. We will refer to $d_{E}$ as the Euclidean metric. The results in [D1] show that provided (2) holds both sets $\mathcal{C}$ and $\mathcal{B}$ are of first Baire category on $\left(\mathcal{M}, d_{E}\right)$. Moreover, we also have result on the Lebesgue measure of the sets $\varphi(\mathcal{C})$ and $\varphi(\mathcal{B})$. The measure of these set is either 0 or 1 (depending on the given sequence $\left(b_{n}\right)_{n \in \mathbb{N}}$ ). These results there are actually valid for any sequence $\left(b_{n}\right)_{n \in \mathbb{N}}$ from a Hilbert space $H$.

In the paper [D2] we conducted a similar study for Frèchet $\left(d_{F}\right)$ and Baire $\left(d_{B}\right)$ metric. Here

$$
\begin{aligned}
& d_{F}(a, b)=\sum_{n=1}^{\infty} \frac{1}{2^{n}} \frac{\left|a_{n}-b_{n}\right|}{1+\left|a_{n}-b_{n}\right|}, \quad \text { Frèchet metric, } \\
& d_{B}(a, b)=\left\{\begin{array}{ll}
\frac{1}{\min \left\{n \in \mathbb{N} ; a_{n} \neq b_{n}\right\}} & \text { for } a \neq b, \\
0 & \text { for } a=b,
\end{array}\right. \text { Baire metric. }
\end{aligned}
$$


The major fault of the previous metrics comes from the fact that they do not control the 'tail' of a sequence $\left(a_{n}\right)_{n \in \mathbb{N}}$. This means that given any sequence $\left(a_{n}\right)_{n \in \mathbb{N}}$ we can alter its tail completely arbitrarily. The new sequence we get can be arbitrarily close the original one. It is however the 'tail' of the sequence $\left(a_{n}\right)_{n \in \mathbb{N}}$ that decides whether the corresponding series (1) converges or not. This means that the metrics mentioned are not optimal for studying convergence of (1).

It would be therefore desirable to find a metric on the space $\{0,1\}^{\mathbb{N}}$ which does not possess this fault. We have managed to find such a metric. The precise definition is given bellow. This metric turns out to have some advantages over the previous ones and gives us better tool for study the problem outlined above.

\section{The Definition of the D-metric}

Here we give the definition of the D-metric. Although we mainly want to study the space $\{0,1\}^{\mathbb{N}}$, the definition is valid in any Banach space. Let $X$ be a Banach space and $M \subset X$ be a closed bounded subset. $M$ with the metric inherited from $X$ is a complete metric space. For the sequences

$$
a=\left(a_{1}, a_{2}, a_{3}, \ldots\right) \text {, and } b=\left(b_{1}, b_{2}, b_{3}, \ldots\right),
$$

where all $a_{n}$ and $b_{n}$ are from $M$, (i.e. $a, b \in M^{\mathbb{N}}$ ) we define the D-metric $d_{D}(a, b)$ by

$$
\begin{aligned}
d_{D}(a, b)=\sup \left\{\left|\frac{a_{1}-b_{1}}{1}\right|,\left|\frac{a_{1}+a_{2}-b_{1}-b_{2}}{2}\right|\right. & \\
& \left.\left|\frac{a_{1}+a_{2}+a_{3}-b_{1}-b_{2}-b_{3}}{3}\right|, \ldots\right\} .
\end{aligned}
$$

Here |.| means the norm in the space $X$. Clearly, the boundedness of the set $M$ guarantees that the number $d_{D}(a, b)$ is always well defined and finite. We can also lift the condition of boundedness of $M$ if we allow $d_{D}$ to take infinite values. Or if we want to have finiteness of the metric we simply put $\widetilde{d_{D}}(a, b)=\min \left\{d_{D}(a, b), 1\right\}$. The next theorem justifies our definition.

Theorem 2.1. Let $X$ is a Banach space, $M \subset X$ a closed bounded set. For any $a, b \in M^{\mathbb{N}}$ if we define the function $d_{D}(a, b)$ by $(3)$, then $d_{D}$ is a metric on $M^{\mathbb{N}}$ and $\left(M^{\mathbb{N}}, d_{D}\right)$ is a complete metric space. The same is true of the metric $\widetilde{d_{D}}=\min \left\{d_{D}, 1\right\}$ even if we drop the condition of boundedness of the set $M$.

Proof. The proof that $d_{D}$ is a metric is trivial. Let us for example check the triangle inequality for $d_{D}$. Take $a, b, c \in M^{\mathbb{N}}$, we want to demonstrate that

$$
d_{D}(a, c) \leq d_{D}(a, b)+d_{D}(b, c) .
$$


By the definition of $d_{D}$ for any $k \in \mathbb{N}$ we have

$$
\begin{aligned}
& \left|\frac{a_{1}+a_{2}+\cdots+a_{k}-b_{1}-b_{2}-\cdots-b_{k}}{k}\right| \leq d_{D}(a, b) \text { and } \\
& \left|\frac{b_{1}+b_{2}+\cdots+b_{k}-c_{1}-c_{2}-\cdots-c_{k}}{k}\right| \leq d_{D}(b, c) .
\end{aligned}
$$

Hence by the triangle inequality

$$
\begin{aligned}
& \left|\frac{a_{1}+a_{2}+\cdots+a_{k}-c_{1}-c_{2}-\cdots-c_{k}}{k}\right| \\
\leq & \left|\frac{a_{1}+a_{2}+\cdots+a_{k}-b_{1}-b_{2}-\cdots-b_{k}}{k}\right| \\
& +\left|\frac{b_{1}+b_{2}+\cdots+b_{k}-c_{1}-c_{2}-\cdots-c_{k}}{k}\right| \leq d_{D}(a, b)+d_{D}(b, c) .
\end{aligned}
$$

Taking supremum over all integers $k$ yields (4).

Now we show that $d_{D}$ is a complete metric space. Assume that $\left(A_{n}\right)_{n \in \mathbb{N}}$ is a Cauchy sequence in $M^{\mathbb{N}}$. We will write $A_{n}$ as $A_{n}=\left(a_{n 1}, a_{n 2}, a_{n 3}, \ldots\right)$ where $a_{n i}$ belongs to $M$. From the definition of $d_{D}$ metric it follows that $\left|a_{n 1}-a_{m 1}\right| \leq d_{D}\left(A_{n}, A_{m}\right)$ which means that $\left(a_{n 1}\right)_{n \in \mathbb{N}}$ is a Cauchy sequence in $M$. Similarly for $a_{n 2}$ we get

$$
\begin{gathered}
\frac{\left|a_{n 2}-a_{m 2}\right|}{2}-\frac{\left|a_{n 1}-a_{m 1}\right|}{2} \leq \frac{\left|a_{n 1}+a_{n 2}-a_{m 1}-a_{m 2}\right|}{2} \leq d_{D}\left(A_{n}, A_{m}\right) \\
\left|a_{n 2}-a_{m 2}\right| \leq\left|a_{n 1}-a_{m 1}\right|+2 d_{D}\left(A_{n}, A_{m}\right)
\end{gathered}
$$

By using induction and similar arguments we obtain that for all $i \in \mathbb{N}$, $\left(a_{n i}\right)_{n \in \mathbb{N}}$ is a Cauchy sequence. Therefore we can put $a_{i}=\lim _{n \rightarrow \infty} a_{n i}$. Since $M$ is a closed set, $a_{i} \in M$ and hence the sequence $A=\left(a_{1}, a_{2}, a_{3}, \ldots\right)$ belongs to $M^{\mathbb{N}}$. We want to show $A=\lim _{n \rightarrow \infty} A_{n}$. Let $\varepsilon>0$. Then there is a $k \in \mathbb{N}$ such that for all $m, n \geq k d_{D}\left(A_{n}, A_{m}\right)<\varepsilon$. Then

$$
\begin{aligned}
& \left|\frac{a_{1}+a_{2}+\cdots+a_{i}-a_{n 1}-a_{n 2}-\cdots-a_{n i}}{i}\right| \\
& =\lim _{m \rightarrow \infty}\left|\frac{a_{m 1}+a_{m 2}+\cdots+a_{m i}-a_{n 1}-a_{n 2}-\cdots-a_{n i}}{i}\right| \leq \varepsilon .
\end{aligned}
$$

Hence we have that $d\left(A, A_{n}\right) \leq \varepsilon$ for any $n \geq k$ giving us the desired result. The case of the metric $\widetilde{d_{D}}$ is analogous.

The next lemma gives us partial insight into the topology of the space $\left(M^{\mathbb{N}}, d_{D}\right)$. 
Lemma 2.2. Consider the complete metric space $\left(M^{\mathbb{N}}, d_{D}\right)$. Let $k$ be a positive integer, $c$ a real number and $\left(\alpha_{1}, \alpha_{2}, \ldots \alpha_{k}\right)$ a finite sequence of real numbers. Let

$$
\begin{aligned}
& \mathcal{S}_{o}=\left\{\left(a_{n}\right)_{n \in \mathbb{N}} \in M^{\mathbb{N}} ;\left|\alpha_{1} a_{1}+\alpha_{2} a_{2}+\cdots+\alpha_{k} a_{k}\right|>c\right\}, \\
& \mathcal{S}_{i}=\left\{\left(a_{n}\right)_{n \in \mathbb{N}} \in M^{\mathbb{N}} ;\left|\alpha_{1} a_{1}+\alpha_{2} a_{2}+\cdots+\alpha_{k} a_{k}\right|<c\right\} .
\end{aligned}
$$

Then both sets $\mathcal{S}_{o}$ and $\mathcal{S}_{i}$ are open subsets of the metric space $\left(M^{\mathbb{N}}, d_{D}\right)$.

Proof. First consider one special case: $\alpha_{1}=\alpha_{2}=\cdots=\alpha_{k}=0$. Clearly, for $c \geq 0$ we have $S_{o}=\emptyset$ and $S_{i}=M^{\mathbb{N}}$ and for $c<0 S_{o}=M^{\mathbb{N}}$ and $S_{i}=\emptyset$. So the lemma holds.

Now consider all remaining cases. We will prove this lemma only for the set $S_{o}$ since the proof for the other set is similar. Take any $\left(a_{n}\right)_{n \in \mathbb{N}} \in S_{o}$. We need to show that there is an $\varepsilon>0$ such that each sequence $\left(b_{n}\right)_{n \in \mathbb{N}}$ whose distance from $\left(a_{n}\right)_{n \in \mathbb{N}}$ is less than $\varepsilon$ is also in $S_{o}$. Let

$$
\begin{aligned}
\alpha & =\max \left\{\left|\alpha_{1}\right|,\left|\alpha_{2}\right|, \ldots\left|\alpha_{k}\right|\right\}>0, \\
\delta & =\left|\alpha_{1} a_{1}+\alpha_{2} a_{2}+\cdots+\alpha_{k} a_{k}\right|-c .
\end{aligned}
$$

Pick $\varepsilon>0$ so small that $d_{D}(a, b)<\varepsilon \Longrightarrow\left|a_{i}-b_{i}\right|<\frac{\delta}{k \alpha} \quad$ for $i=1,2, \ldots, k$. Such $\varepsilon$ always exists. Now we have

$$
\begin{aligned}
& \left|\alpha_{1} b_{1}+\alpha_{2} b_{2}+\cdots+\alpha_{k} b_{k}\right| \\
= & \left|\alpha_{1} a_{1}+\alpha_{2} a_{2}+\cdots+\alpha_{k} a_{k}+\alpha_{1}\left(b_{1}-a_{1}\right)+\alpha_{2}\left(b_{2}-a_{2}\right)+\cdots+\alpha_{k}\left(b_{k}-a_{k}\right)\right| \\
\geq & \left|\alpha_{1} a_{1}+\alpha_{2} a_{2}+\cdots+\alpha_{k} a_{k}\right|-\left|\alpha_{1}\right|\left|b_{1}-a_{1}\right|-\left|\alpha_{2}\right|\left|b_{2}-a_{2}\right|-\cdots-\left|\alpha_{k}\right|\left|b_{k}-a_{k}\right| \\
> & c+\delta-k \alpha \frac{\delta}{k \alpha}=c .
\end{aligned}
$$

Hence the sequence $\left(b_{n}\right)_{n \in \mathbb{N}}$ is in $S_{o}$.

If we take $M=\{0,1\} \subset \mathbb{R}$, we can state the previous lemma for this space.

Corollary 2.3. Consider the complete metric space $\left(\{0,1\}^{\mathbb{N}}, d_{D}\right)$. Let $k$ be a positive integer, $c$ a real number and $\left(\alpha_{1}, \alpha_{2}, \ldots \alpha_{k}\right)$ a finite sequence of real numbers. Let $\mathcal{S}_{+}$and $\mathcal{S}_{-}$be the sets

$$
\begin{aligned}
& \mathcal{S}_{+}=\left\{\left(a_{n}\right)_{n \in \mathbb{N}} \in\{0,1\}^{\mathbb{N}} ; \alpha_{1} a_{1}+\alpha_{2} a_{2}+\cdots+\alpha_{k} a_{k}>c\right\}, \\
& \mathcal{S}_{-}=\left\{\left(a_{n}\right)_{n \in \mathbb{N}} \in\{0,1\}^{\mathbb{N}} ; \alpha_{1} a_{1}+\alpha_{2} a_{2}+\cdots+\alpha_{k} a_{k}<c\right\} .
\end{aligned}
$$

Then both sets $\mathcal{S}_{+}$and $\mathcal{S}_{-}$are open subsets of the metric space $\left(\{0,1\}^{\mathbb{N}}, d_{D}\right)$.

It might also be interesting to compare all of these metrics on the space $\{0,1\}^{\mathbb{N}}$. We get $d_{D} \geq d_{B} \geq d_{F} \geq \frac{1}{2} d_{E}$. Since none of these inequalities 
can be reversed, these metrics are not equivalent. It is known however that the Baire and Frèchet metrics generate the same topology on $\{0,1\}^{\mathbb{N}}$. The same is not true about the D-metric whose topology is different. This follows for example from the fact that the sequence $\left(A_{n}\right)_{n \in \mathbb{N}}$ defined by $A_{n}=$ $(\underbrace{1,1, \ldots, 1}, 0,0, \ldots), n=1,2,3, \ldots$ is Cauchy in $d_{F}, d_{B}$ and $d_{E}$ but not in $d_{D}$.

The above inequalities also means that the space $\left(\{0,1\}^{\mathbb{N}}, d_{D}\right)$ is the richest one; i.e., it has more open sets than the other metric spaces considered.

\section{Study of Interesting Properties of the D-Metric}

In this section we extend the simple results about the D-metric from the previous section. In the entire section we assume that

$M \subset X$ is closed and bounded and contains a nonzero element.

We want to define one very important notion.

Definition 3.1. Let $M \subset X$ be a bounded set of a Banach space $X$. Let $\left(x_{n}\right)_{\in \mathbb{N}}$ be a given sequence of elements of $M$. let

$$
\begin{gathered}
\mathcal{A}=\left\{a \in X ; \text { there is an increasing sequence }\left(n_{k}\right)_{k \in \mathbb{N}}\right. \text { of integers } \\
\text { such that } \left.\lim _{k \rightarrow \infty}^{*} \frac{x_{1}+x_{2}+\cdots+x_{n_{k}}}{n_{k}}=a\right\} .
\end{gathered}
$$

We will call $\mathcal{A}$ 'the set of limit averages in the sequence $\left(x_{n}\right)_{n \in \mathbb{N}}$ '. (Here lim denotes the limit in the weak topology of the space $X$.) If for a certain sequence $\left(x_{n}\right)_{n \in \mathbb{N}}$ the set $\mathcal{A}$ has exactly one element for which we have

$$
A\left(\left(x_{n}\right)_{n \in \mathbb{N}}\right)=\lim _{n \rightarrow \infty}^{*} \frac{x_{1}+x_{2}+\cdots+x_{n}}{n}
$$

we call such an element $A\left(\left(x_{n}\right)_{n \in \mathbb{N}}\right)$ the limit average of the sequence $\left(x_{n}\right)_{n \in \mathbb{N}}$.

Remark. Of course if $X$ is finite dimensional, the weak limit in the definition of $\mathcal{A}$ can be replaced by the limit in the norm of $X$. If the space $X$ is reflexive, the set $\mathcal{A}$ must be nonempty, since any bounded convex set is compact in the weak topology. Note that we used the calligraphic letter $\mathcal{A}$ to denote the set and ordinary letter $A$ to denote the unique weak limit of the Caesaro averages of the sequence $\left(x_{n}\right)_{n \in \mathbb{N}}$ if such limit exists. 
Another way to write the set $\mathcal{A}$ is

$$
\mathcal{A}=\bigcap_{n=1}^{\infty}{\overline{\bigcup_{k=n}^{\infty}\left\{\frac{x_{1}+x_{2}+\cdots+x_{k}}{k}\right\}}}^{w},
$$

where the closure is taken in the weak topology.

In case when $M=\{0,1\} \subset \mathbb{R}$; i.e., $\left(a_{n}\right)_{n \in \mathbb{N}} \in\{0,1\}^{\mathbb{N}}$ we can also define the following notion

Definition 3.2. Let $\left(a_{n}\right)_{n \in \mathbb{N}} \in\{0,1\}^{\mathbb{N}}$. The lower (upper) density of ones in the sequence $\left(a_{n}\right)_{n \in \mathbb{N}}$ are

$$
\begin{aligned}
& h_{L}=\varliminf_{n \rightarrow \infty} \frac{a_{1}+a_{2}+\cdots+a_{n}}{n}, \\
& h_{U}=\varlimsup_{n \rightarrow \infty} \frac{a_{1}+a_{2}+\cdots+a_{n}}{n},
\end{aligned}
$$

respectively. If those numbers are equal, we talk about the density of ones in the sequence $\left(a_{n}\right)_{n \in \mathbb{N}}$ and denote it by $h$ or more precisely by $h\left(\left(a_{n}\right)_{n \in \mathbb{N}}\right)$.

The number $h\left(\left(a_{n}\right)_{n \in \mathbb{N}}\right)$ gives the 'ratio' between zeros and ones in the sequence $\left(a_{n}\right)_{n \in \mathbb{N}}$. See [D3] for a more detailed treatment. The connection between the notion of the density of ones in the sequence $\left(a_{n}\right)_{\in \mathbb{N}} \in\{0,1\}^{\mathbb{N}}$ and the set $\mathcal{A}$ defined above is the following.

Take the set $\mathrm{M}$ to be $M=\{0,1\}$ and let $x_{n}=a_{n}$. If $\mathcal{A}$ is defined for $\left(x_{n}\right)_{n \in \mathbb{N}}$ as in Definition 3.1, then we have $h_{U}\left(\left(a_{n}\right)_{n \in \mathbb{N}}\right)=\sup \mathcal{A}$, and $h_{L}\left(\left(a_{n}\right)_{n \in \mathbb{N}}\right)=\inf \mathcal{A}$. In particular $h\left(\left(a_{n}\right)_{n \in \mathbb{N}}\right)=\frac{1}{2}$ is equivalent to the fact that the set $\mathcal{A}$ contains only one element and $A\left(\left(x_{n}\right)_{n \in \mathbb{N}}\right)=\frac{1}{2}$.

Now we return to the general case where $X$ is a Banach space. In order to be able to get results in this general setting we use continuous projections of $X$ onto its one dimensional subspaces. This considerably reduces the difficulty of our analysis. It is a simple exercise about Banach spaces to prove following.

Proposition 3.3. Let $L \subset X$ be any 1-dimensional subspace of $X$. Then there is a continuous projection $P: X \rightarrow L$ for which $P(L)=L$. Moreover, this projection can be chosen so that its norm is one.

Proof. Since $L$ is a one dimensional subspace, it is spanned by a certain element $z \in X$ of a unit norm; i.e., $L=\{t z ; t \in \mathbb{R}\}$. If we define a mapping $S: L \rightarrow \mathbb{R}$ by $S(t z)=t$, for all $t \in \mathbb{R}$, then the map $S$ is a linear isomorphism between the subspace $L$ and the real line $\mathbb{R}$. Therefore the norm of this map is one. Since $L \subset X$ by the Hahn-Banach theorem there is a continuous linear extension $\widetilde{S}: X \rightarrow \mathbb{R}$ of $S: L \rightarrow \mathbb{R}$ with norm one. Then $P=S^{-1} \circ \widetilde{S}$ is the desired projection. 
For convenience in everything that follows we identify the one dimensional subspace $L$ defined above with the real line $\mathbb{R}$; i.e., we do not distinguish between the maps $P$ and $\widetilde{S}$ since via this identification their range space is same. For this reason we can consider $P$ as a map in $X^{\prime}$ the dual of $X$.

So for any linear and bounded map $P: X \rightarrow \mathbb{R}$ we can consider the set $\mathcal{A}$ from Definition 3.1 for the sequence $\left(x_{n}\right)_{n \in \mathbb{N}} \in M^{\mathbb{N}}$ and also $\mathcal{A}^{P}$ - the set from Definition 3.1 for the corresponding sequence $\left(P\left(x_{n}\right)\right)_{n \in \mathbb{N}} \in \mathbb{R}^{\mathbb{N}}$. What we see is that

$$
P(\mathcal{A}) \subset \mathcal{A}^{P}
$$

We cannot guarantee equality here (at least not for spaces $X$ which are not reflexive) but (6) will suffice for our purposes. This indicates our next steps. We concentrate on the setting when $M \subset \mathbb{R}$, which is not so difficult, and then generalize our results using (6).

For $\left(x_{n}\right)_{n \in \mathbb{N}} \in \mathbb{R}^{\mathbb{N}}$ consider two numbers

$$
A_{L}\left(\left(x_{n}\right)_{n \in \mathbb{N}}\right)=\inf \mathcal{A}, \text { and } A_{U}\left(\left(x_{n}\right)_{n \in \mathbb{N}}\right)=\sup \mathcal{A} .
$$

If $A_{L}=A_{U}$ their common value is exactly the number $A\left(\left(x_{n}\right)_{n \in \mathbb{N}}\right)$ from Definition 3.1. Now we are ready to prove this important lemma.

Lemma 3.4. Assume that (5) holds. Let $\left(x_{n}\right)_{n \in \mathbb{N}} \in M^{\mathbb{N}}$ be a given sequence for which there is a $P \in X^{\prime}$ such that $\varepsilon=A_{U}\left(\left(P\left(x_{n}\right)\right)_{n \in \mathbb{N}}\right)-A_{L}\left(\left(P\left(x_{n}\right)\right)_{n \in \mathbb{N}}\right)$ is positive. Then for any sequence $\left(y_{n}\right)_{n \in \mathbb{N}}$ in an open ball (in the D-metric) of radius $\frac{\varepsilon}{2|P|}\left(|P|\right.$ is the norm of $P$ in $\left.X^{\prime}\right)$ centered at $\left(x_{n}\right)_{n \in \mathbb{N}}$ we have

$$
A_{U}\left(\left(P\left(y_{n}\right)\right)_{n \in \mathbb{N}}\right)-A_{L}\left(\left(P\left(y_{n}\right)\right)_{n \in \mathbb{N}}\right)>0 .
$$

Proof. Take any sequence $\left(y_{n}\right)_{n \in \mathbb{N}}$ for which $d_{D}(x, y)<\frac{\varepsilon}{2|P|}$. There is a $\delta>0$ such that $d_{D}(x, y)<\frac{\varepsilon}{2|P|}-\frac{\delta}{|P|}$.

For any $n \in \mathbb{N}$ we have

$$
\begin{aligned}
& P\left(\frac{y_{1}+y_{2}+\cdots+y_{n}}{n}\right) \\
\leq & P\left(\frac{x_{1}+x_{2}+\cdots+x_{n}}{n}\right)+\left|P\left(\frac{y_{1}+y_{2}+\cdots+y_{n}-x_{1}-x_{2}-\cdots-x_{n}}{n}\right)\right| \\
\leq & P\left(\frac{x_{1}+x_{2}+\cdots+x_{n}}{n}\right)+\frac{\varepsilon}{2}-\delta .
\end{aligned}
$$


Therefore

$A_{L}\left(\left(P\left(y_{n}\right)\right)_{n \in \mathbb{N}}\right)=\varliminf_{n \rightarrow \infty} P\left(\frac{y_{1}+y_{2}+\cdots+y_{n}}{n}\right) \leq A_{L}\left(\left(P\left(x_{n}\right)\right)_{n \in \mathbb{N}}\right)+\frac{\varepsilon}{2}-\delta$.

Also

$A_{U}\left(\left(P\left(y_{n}\right)\right)_{n \in \mathbb{N}}\right)=\varlimsup_{n \rightarrow \infty} P\left(\frac{b_{1}+b_{2}+\cdots+b_{n}}{n}\right) \geq A_{U}\left(\left(P\left(x_{n}\right)\right)_{n \in \mathbb{N}}\right)-\frac{\varepsilon}{2}+\delta$.

Together we get

$$
\begin{aligned}
& A_{U}\left(\left(P\left(y_{n}\right)\right)_{n \in \mathbb{N}}\right)-A_{L}\left(\left(P\left(y_{n}\right)\right)_{n \in \mathbb{N}}\right) \geq \\
\geq & A_{U}\left(\left(P\left(x_{n}\right)\right)_{n \in \mathbb{N}}\right)-A_{L}\left(\left(P\left(x_{n}\right)\right)_{n \in \mathbb{N}}\right)-\varepsilon+2 \delta=2 \delta>0 .
\end{aligned}
$$

Now we can establish one quite interesting result:

Theorem 3.5. Let $X$ be a Banach space. Assume that for $M \subset X(5)$ holds and $M$ contains at least two distinct elements. Let $F \subset X$ be a closed subset of the space $X$. Then the set

$$
\mathcal{T}_{F}=\left\{\left(x_{n}\right)_{n \in \mathbb{N}} \in M^{\mathbb{N}} ; A\left(x_{n}\right)_{n \in \mathbb{N}} \text { exists and } A\left(\left(x_{n}\right)_{n \in \mathbb{N}}\right) \in F\right\}
$$

is closed and nowhere dense in the complete metric space $\left(M^{\mathbb{N}}, d_{D}\right)$. (The definition of $A\left(x_{n}\right)_{n \in \mathbb{N}}$ is given in 3.1.)

Proof. First we establish that the set $\mathcal{T}_{F}$ is closed. We look at the complement of this set.

$$
\begin{aligned}
G=M^{\mathbb{N}} \backslash \mathcal{T}_{F}=\left\{\left(x_{n}\right)_{n \in \mathbb{N}} ;\right. & \text { the weak limit } A\left(\left(x_{n}\right)_{n \in \mathbb{N}}\right) \\
& \text { does not exist or exists but is not in } F\} .
\end{aligned}
$$

We want to show that this set $G$ is an open set in our metric space. Pick any $\left(x_{n}\right)_{n \in \mathbb{N}} \in G$. We want to find a $\varepsilon>0$ such that all sequences in a ball of radius $\varepsilon$ centered at $\left(x_{n}\right)_{n \in \mathbb{N}}$ are also in $G$. There are two possibilities for our sequence. Either $A=A\left(x_{n}\right)_{n \in \mathbb{N}}$ does not exist or $A \notin F$.

First consider the case $A$ does not exist. Then there is a $P \in X^{\prime}$ such that the sequence $P\left(\frac{x_{1}+x_{2}+\cdots+x_{n}}{n}\right)$ for $n=1,2, \ldots$ does not converge and therefore $A_{U}\left(\left(P\left(x_{n}\right)\right)_{n \in \mathbb{N}}\right)-A_{L}\left(\left(P\left(x_{n}\right)\right)_{n \in \mathbb{N}}\right)>0$. Lemma 3.4 takes care of the rest.

Consider now the other case; i.e., let $A\left(\left(x_{n}\right)_{n \in \mathbb{N}}\right)$ exists for $\left(x_{n}\right)_{n \in \mathbb{N}} \in G$, but $A \notin F$. Since the set $F$ is closed in $X$, there is a $\varepsilon>0$ such that for all $\widetilde{A}$ we have $|\widetilde{A}-A|<2 \varepsilon \Longrightarrow \widetilde{A} \notin F$. Take any sequence $\left(y_{n}\right)_{n \in \mathbb{N}}$ for 
which $d(x, y)<\varepsilon$. If $\widetilde{A}=A\left(\left(y_{n}\right)_{n \in \mathbb{N}}\right)$ does not exist, we are done since then necessarily $\left(y_{n}\right)_{n \in \mathbb{N}} \in G$. On the other hand, if $\widetilde{A}$ exists then we will show that $|\widetilde{A}-A|<2 \varepsilon$, and thus $\left(y_{n}\right)_{n \in \mathbb{N}} \in G$. To see this, we assume the contrary; i.e., $|\widetilde{A}-A| \geq 2 \varepsilon$. Let $z=\widetilde{A}-A$. Consider the line $L=\{t z ; t \in \mathbb{R}\}$ spanned by $z$. By Proposition 3.3 there is a projection $O$ of $X$ onto $L$ with norm one. If we identify $L$ with $\mathbb{R}$ via the map $T: L \rightarrow \mathbb{R}$ also of the norm one, for $P=T \circ O \in X^{\prime}$ we get

$$
|P(\widetilde{A})-P(A)|=|P(z)|=|O(z)|=|z|=|\widetilde{A}-A| \geq 2 \varepsilon .
$$

Clearly, the norm of the map $P$ is also one. Then

$$
\begin{aligned}
|P(\widetilde{A})-P(A)| & =\lim _{n \rightarrow \infty}\left|P\left(\frac{y_{1}+y_{2}+\cdots+y_{n}-x_{1}-x_{2}-\cdots-x_{n}}{n}\right)\right| \\
& \leq|P| \varepsilon=\varepsilon .
\end{aligned}
$$

This contradicts (8) and therefore $|\widetilde{A}-A| \geq 2 \varepsilon$ cannot hold. This completes the proof of the first part of the theorem.

Now we want to establish that the set, $\mathcal{T}_{F}$, defined in (7) is nowhere dense. Let $\left(x_{n}\right)_{n \in \mathbb{N}}$ be any sequence that belongs to $\mathcal{T}_{F}$ and $\varepsilon>0$ be a positive number. We want to show that in a ball of radius $\varepsilon$ centered at $\left(x_{n}\right)_{n \in \mathbb{N}}$ there is a sequence $\left(y_{n}\right)_{n \in \mathbb{N}} \notin \mathcal{T}_{F}$. We do this by showing that for certain $P \in X^{\prime}$

$$
A_{L}\left(\left(P\left(y_{n}\right)\right)_{n \in \mathbb{N}}\right)<A_{U}\left(\left(P\left(y_{n}\right)\right)_{n \in \mathbb{N}}\right) .
$$

Constructing the sequence $\left(y_{n}\right)_{n \in \mathbb{N}}$ is very simple. It will be defined by slightly modifying $\left(x_{n}\right)_{n \in \mathbb{N}}$. For given $\varepsilon>0$ we find a positive integer $k$ such that $\frac{1}{2^{k}}<\frac{\varepsilon}{2 K}$, where $K=\operatorname{diam}(M)$ is the diameter of the set $M$. Since the set $M$ contains at least two elements we can find $P \in X^{\prime}$ of norm 1 such that for some $m_{0}, m_{1} \in M P\left(m_{1}\right)>0$ and $P\left(m_{0}\right)<P\left(m_{1}\right)$. Let $c=\frac{1}{2}\left(P\left(m_{0}\right)+P\left(m_{1}\right)\right)$. For each $j \geq k+1$ there are at least $2^{j-k}$ numbers bigger or equal to $c$ or at least $2^{j-k}$ numbers less than $c$ among

$$
P\left(x_{2^{j}+1}\right), P\left(x_{2^{j}+2}\right), P\left(x_{2^{j}+3}\right), \ldots, P\left(x_{2^{j}+2^{j}}\right) .
$$

Hence we can sort out all integers $j \geq k+1$ into two increasing sequences of integers $u_{1}<u_{2}<u_{3}<\ldots$ and $v_{1}<v_{2}<v_{3}<\ldots$ such that an integer $j \geq k+1$ is in the sequence $\left(u_{n}\right)_{n \in I_{1}}$ if there are at least $2^{j-k}$ numbers bigger or equal to $c$ among $P\left(x_{2^{j}+1}\right), P\left(x_{2^{j}+2}\right), \ldots, P\left(x_{2^{j}+2^{j}}\right)$, and in the sequence $\left(v_{n}\right)_{n \in I_{2}}$ otherwise. Of course it is possible that one of these two sequences is finite but at least one of them must be infinite. Without loss of generality we 
assume that this is true for the sequence $\left(u_{n}\right)_{n \in I_{1}}$. So the set of indices $I_{1}$ is just $\mathbb{N}$.

Now we begin to define our sequence $\left(y_{n}\right)_{n \in \mathbb{N}}$. First we put

$$
y_{n}= \begin{cases}x_{n}, & \text { for } n=1,2, \ldots, 2^{k+1}, \\ x_{n}, & \text { if } 2^{v_{j}}<n \leq 2^{v_{j}+1} \text { for some } j \in I_{2} .\end{cases}
$$

It remains to define $\left(y_{n}\right)_{n \in \mathbb{N}}$ for $2^{u_{j}}<n \leq 2^{u_{j}+1}, j=1,2,3, \ldots$ From the definition of the number $u_{j}$ there are indices

$$
2^{u_{j}}+1 \leq n_{1}<n_{2}<n_{3}<\ldots n_{l_{j}}<2^{u_{j}+1},
$$

where $l_{j}=2^{u_{j}-k}$ such that the numbers $P\left(x_{n_{1}}\right), P\left(x_{n_{2}}\right), \ldots, P\left(x_{n_{l_{j}}}\right)$ are bigger or equal to $c$. Now we consider the number

$$
\left|\frac{x_{1}+x_{2}+\cdots+x_{2^{u_{j}}}-y_{1}-y_{2}-\cdots-y_{2^{u_{j}}}}{2^{u_{j}}}\right| .
$$

If this number is less than $\alpha_{j}$ (defined later) we put

$$
y_{n}= \begin{cases}x_{n}, & 2^{u_{j}}<n \leq 2^{u_{j}+1} \text { and } n \notin\left\{n_{1}, n_{2}, \ldots, n_{l_{j}}\right\}, \\ m_{0}, & n \in\left\{n_{1}, n_{2}, \ldots, n_{l_{j}}\right\} .\end{cases}
$$

Otherwise, we just take $y_{n}=x_{n}, 2^{u_{j}}<n \leq 2^{u_{j}+1}$.

The sequence $\left(\alpha_{j}\right)_{j \in \mathbb{N}}$ is defined by

$$
\alpha_{j}= \begin{cases}\frac{\varepsilon}{2}-\frac{K}{2^{k}}, & \text { for } j=1, \\ \alpha_{j-1}, & \text { if }(10) \text { for } j-1 \text { is bigger or equal to } \alpha_{j-1}, \\ \frac{\alpha_{j-1}}{2}, & \text { if }(10) \text { for } j-1 \text { is less than } \alpha_{j-1} .\end{cases}
$$

Since the sequence $\left(\alpha_{j}\right)_{j \in \mathbb{N}}$ is constant as long as (10) is greater of equal to $\alpha_{j}$, eventually for sufficiently large $j(10)$ will become smaller than $\alpha_{j}$. This means that $\lim _{j \rightarrow \infty} \alpha_{j}=0$.

Now the sequence $\left(y_{n}\right)_{n \in \mathbb{N}}$ is defined everywhere. It remains to show that this sequence is $\varepsilon$-close to $\left(x_{n}\right)_{n \in \mathbb{N}}$ and that (9) holds. Let us first demonstrate (9). For each $j$ for which (10) is less that $\alpha_{j}$ we have changed exactly $2^{u_{j}-k}$ terms of the sequence $\left(x_{n}\right)_{n \in \mathbb{N}}$. Therefore we have

$$
\begin{aligned}
& \frac{P\left(x_{1}\right)+P\left(x_{2}\right)+\cdots+P\left(x_{2^{u_{j}+1}}\right)-P\left(y_{1}\right)-P\left(y_{2}\right)-\cdots-P\left(y_{2^{u_{j}+1}}\right)}{2^{u_{j}+1}} \\
\geq & \frac{2^{u_{j}-k} \frac{P\left(m_{1}\right)-P\left(m_{0}\right)}{2}}{2^{u_{j}+1}} \geq \frac{P\left(m_{1}\right)-P\left(m_{0}\right)}{2^{k+2}} .
\end{aligned}
$$


From this we deduce

$$
A_{U}\left(\left(P\left(x_{n}\right)\right)_{n \in \mathbb{N}}\right) \geq A_{L}\left(\left(P\left(y_{n}\right)\right)_{n \in \mathbb{N}}\right)+\frac{P\left(m_{1}\right)-P\left(m_{0}\right)}{2^{k+2}} .
$$

On the other hand since (10) is less than $\alpha_{j}$ and this sequence goes to zero, we get $A_{L}\left(\left(P\left(x_{n}\right)\right)_{n \in \mathbb{N}}\right) \leq A_{U}\left(\left(P\left(y_{n}\right)\right)_{n \in \mathbb{N}}\right)$. Together, since $A_{U}\left(\left(P\left(x_{n}\right)\right)_{n \in \mathbb{N}}\right)=$ $A_{L}\left(\left(P\left(x_{n}\right)\right)_{n \in \mathbb{N}}\right)=P\left(A\left(\left(x_{n}\right)_{n \in \mathbb{N}}\right)\right)$ we get the desired conclusion.

$$
A_{U}\left(\left(P\left(y_{n}\right)\right)_{n \in \mathbb{N}}\right)-A_{L}\left(\left(P\left(y_{n}\right)\right)_{n \in \mathbb{N}}\right) \geq \frac{P\left(m_{1}\right)-P\left(m_{0}\right)}{2^{k+2}}>0 .
$$

To see that both sequences are close to each other in $d_{D}$ metric let us evaluate

$$
\left|\frac{y_{1}+y_{2}+y_{3}+\cdots+y_{n}-x_{1}-x_{2}-x_{3}-\cdots-x_{n}}{n}\right| .
$$

We can see that it suffices to look at $n \in \mathbb{N}$ between $2^{u_{j}}<n \leq 2^{u_{j}+1}$, for some $j=1,2,3, \ldots$. This is because only for such $n$ it could happen that $x_{n} \neq y_{n}$, which means the numerator of the quotient (11) could increase. Assume therefore that we have a index $j$ for which (10) is less than $\alpha_{j}$. Then for any $2^{u_{j}}<n \leq 2^{u_{j}+1}$ we get

$$
\begin{aligned}
&\left|\frac{y_{1}+y_{2}+\cdots+y_{n}-x_{1}-x_{2}-\cdots-x_{n}}{n}\right| \\
& \leq\left|\frac{y_{1}+y_{2}+\cdots+y_{2^{u_{j}}}-x_{1}-x_{2}-\cdots-x_{2^{u_{j}}}}{2^{u_{j}}}\right| \\
&+\left|\frac{y_{2^{u_{j}}+1}+y_{2^{u_{j}}+2}+\cdots+y_{2^{u_{j}+1}}-x_{2^{u_{j}}+1}-x_{2^{u_{j}}+2}-\cdots-x_{2^{u_{j}+1}}}{2^{u_{j}}}\right| \\
&<\frac{\varepsilon}{2}-\frac{K}{2^{k}}+\frac{2^{u_{j}-k}}{2^{u_{j}}} K=\frac{\varepsilon}{2} .
\end{aligned}
$$

From this $d_{D}(a, b)<\varepsilon$ follows immediately. So our theorem holds.

A careful look at the proof of the previous theorem reveals that we have much more than just ' $\mathcal{T}_{F}$ is nowhere dense'. Actually, we established that the set $\mathcal{T}_{F}$ is porous. Recall the definition of a porous set.

Definition 3.6. Let $E$ be a set in a metric space. Given the number $c \in(0,1]$ we say that a point $x_{0} \in E$ is a $c$-porosity point of the set $E$ if there exists a sequence of open balls $B_{k}$ with radius $r_{k} \rightarrow 0$ centered at $x_{0}$ such that for each $k$ there is a ball $G_{k}$ of radius $\rho_{k}$ for which $G_{k} \subset B_{k} \backslash E$ and $\varlimsup_{k \rightarrow \infty} \frac{\rho_{k}}{r_{k}} \geq c$.

We say $x_{0}$ is a porosity point of the set $E_{0}$ provided it is a $c$-porosity point of the set $E_{0}$ for some $c>0$. The set $E$ is said to be porous (c-porous) if any its point is a porosity (c-porosity) point of $E$. 
Remark. If in the definition of a point of $c$-porosity we require that for any ball $B_{r}$ of radius $r>0$ centered at $x_{0}$ there is a ball $G_{r}$ of radius $\rho(r)>0$ such that $G_{r} \subset B_{r} \backslash E$ and $\varliminf_{r \rightarrow 0+} \frac{\rho(r)}{r} \geq c$, then $x_{0}$ is said to be very porous point of $E$. Following the previous definition we can define a very porous and $c$-very porous set, respectively.

Theorem 3.7. Let $X$ be a Banach space. Assume that $M \subset X$ satisfies (5) and contains at least two distinct elements. Let $F \subset X$ be a closed subset of the space $X$. Then the set

$$
\mathcal{T}_{F}=\left\{\left(x_{n}\right)_{n \in \mathbb{N}} \in M^{\mathbb{N}} ; A\left(x_{n}\right)_{n \in \mathbb{N}} \text { exists and } A\left(\left(x_{n}\right)_{n \in \mathbb{N}}\right) \in F\right\}
$$

is closed and c-very porous in the complete metric space $\left(M^{\mathbb{N}}, d_{D}\right)$. The number $c>0$ depends only on the Banach space $X$ and the set $M$.

Proof. This proof makes use of the construction given it the previous theorem. The key is to realize that $P \in X^{\prime}$ picked in the second part of the proof depends only on the chosen set $M$. Choose any $\left(x_{n}\right)_{n \in \mathbb{N}}$ in $\mathcal{T}_{F}$. For any integer $k \geq 2$ if we put $\varepsilon_{k}=\frac{K}{2^{k-2}}$ then $\frac{1}{2^{k}}<\frac{\varepsilon_{k}}{2 K}$. ( $K$ is again the diameter of the set $M)$. According to the previous construction there is a sequence $\left(y_{n}\right)_{n \in \mathbb{N}}$ in a ball of radius less that $\varepsilon_{k}$ such that

$$
A_{U}\left(\left(P\left(y_{n}\right)\right)_{n \in \mathbb{N}}\right)-A_{L}\left(\left(P\left(y_{n}\right)\right)_{n \in \mathbb{N}}\right) \geq \frac{P\left(m_{1}\right)-P\left(m_{0}\right)}{2^{k+2}}>0 .
$$

We take $B_{k}=\left\{\left(z_{n}\right)_{n \in \mathbb{N}} ; d(x, z)<2 \varepsilon_{k}\right\}$, and

$$
G_{k}=\left\{\left(z_{n}\right)_{n \in \mathbb{N}} ; d(y, z)<\frac{1}{2} \frac{P\left(m_{1}\right)-P\left(m_{0}\right)}{2^{k+2}}\right\} .
$$

Since the norm of $P$ in the previous theorem was one, according to Lemma 3.4 any sequence $\left(z_{n}\right)_{n \in \mathbb{N}} \in G_{k}$ is not in the set $\mathcal{T}_{F}$. Therefore we get $G_{k} \subset B_{k} \backslash \mathcal{I}_{F}$. Clearly, if we put now

$$
\begin{aligned}
& r_{k}=r\left(B_{k}\right)=2 \varepsilon_{k}=\frac{K}{2^{k-3}}, \\
& \rho_{k}=r\left(G_{k}\right)=\frac{P\left(m_{1}\right)-P\left(m_{0}\right)}{2^{k+3}},
\end{aligned}
$$

then

$$
\underline{\lim }_{k \rightarrow \infty} \frac{\rho_{k}}{r_{k}} \geq \lim _{k \rightarrow \infty} \frac{\frac{P\left(m_{1}\right)-P\left(m_{0}\right)}{2^{k+3}}}{\frac{K}{2^{k-3}}}=\frac{P\left(m_{1}\right)-P\left(m_{0}\right)}{64 K}>0 .
$$


This proves $c$-porosity. But $\frac{c}{2}$-very porosity of the set $\mathcal{T}_{F}$ follows from this immediately, we just have to realize that for any $r>0$ small there is $k \in \mathbb{N}$ such that

$$
r \geq \varepsilon_{k}>\frac{r}{2}
$$

Hence for such $k, G_{k} \subset G_{r}$. Now, in the definition of very porosity we take $\rho(r)=\rho_{k}$ and get an estimate

$\underline{\lim }_{r \rightarrow 0+} \frac{\rho(r)}{r} \geq \underline{\lim }_{k \rightarrow \infty} \frac{\rho_{k}}{2 \varepsilon_{k}} \geq \lim _{k \rightarrow \infty} \frac{\frac{P\left(m_{1}\right)-P\left(m_{0}\right)}{2^{k+3}}}{2 \frac{K}{2^{k-3}}}=\frac{P\left(m_{1}\right)-P\left(m_{0}\right)}{128 K}>0$.

The theorem above has an important corollary if we take $M=\{0,1\}$.

Corollary 3.8. Let $c \in[0,1]$ be a real number. The set

$$
\mathcal{T}_{c}=\left\{\left(a_{n}\right)_{n \in \mathbb{N}} \in\{0,1\}^{\mathbb{N}} ; h\left(\left(a_{n}\right)_{n \in \mathbb{N}}\right)=c\right\}
$$

is closed, nowhere dense and $\frac{1}{16}$-very porous in the metric space $\left(\{0,1\}^{\mathbb{N}}, d_{D}\right)$. (One can get the constant $\frac{1}{16}$ if we closely follow the proof of Theorem 3.7 for this special case $M=\{0,1\}$. One also gets $\frac{1}{8}$-porosity of the set $\mathcal{T}_{c}$ ).

We can also establish a variant of Corollary 3.8.

Proposition 3.9. Let $0 \leq h_{0} \leq h_{1} \leq 1$ be given numbers. Then the set

$$
\left\{\left(a_{n}\right)_{n \in \mathbb{N}} \in\{0,1\}^{\mathbb{N}} ; h_{0} \leq h_{L}\left(\left(a_{n}\right)_{n \in \mathbb{N}}\right) \leq h_{U}\left(\left(a_{n}\right)_{n \in \mathbb{N}}\right) \leq h_{1}\right\}
$$

is closed in the complete metric space $\left(\{0,1\}^{\mathbb{N}}, d_{D}\right)$.

Since the proof is practically identical with the proof of Theorem 3.6 (making use of Lemma 3.4) we omit it.

\section{Application to Series with Alternating Signs}

Now we are ready to establish several results about the 'size' of the sets $\mathcal{C}$ and $\mathcal{B}$ in the D-metric for the series (1) analogous to those stated for a Frèchet, Baire and Euclidean metrics in [D1] and [D2]. Here we make use of several results which can be found in $[\mathrm{C}],[\mathrm{D} 3],[\mathrm{H}]$ and $[\mathrm{Z}]$. Our first result says when these sets are porous in the metric space $\left(\{0,1\}^{\mathbb{N}}, d_{D}\right)$. 
Theorem 4.1. Let $\left(b_{n}\right)_{n \in \mathbb{N}}$ be a nonincreasing sequence of real numbers for which $\varliminf_{n \rightarrow \infty} n b_{n}>0$ and $\lim _{n \rightarrow \infty} b_{n}=0$. Then the set

$$
\mathcal{C}=\left\{\left(a_{n}\right)_{n \in \mathbb{N}} \in\{0,1\}^{\mathbb{N}} ; \text { series (1) converges }\right\}
$$

is nowhere dense and $\frac{1}{16}$-very porous in the complete metric space $\left(\{0,1\}^{\mathbb{N}}, d_{D}\right)$. If $\lim _{n \rightarrow \infty} n b_{n}=\infty$, then also the set

$$
\mathcal{B}=\left\{\left(a_{n}\right)_{n \in \mathbb{N}} \in\{0,1\}^{\mathbb{N}} ; \exists M>0 \quad \forall k \quad\left|\sum_{n=1}^{k}(-1)^{a_{n}} b_{n}\right| \leq M\right\}
$$

is nowhere dense and $\frac{1}{16}$-very porous in the complete metric space $\left(\{0,1\}^{\mathbb{N}}, d_{D}\right)$. Moreover, this set is of type $F_{\sigma}$ in this space.

Proof. According to [C] or Theorem 3.4 of [D3] if for such sequence $\left(b_{n}\right)_{n \in \mathbb{N}}$ the series

$$
\sum_{n=1}^{\infty}(-1)^{a_{n}} b_{n}
$$

converges, then necessarily $h\left(\left(a_{n}\right)_{n \in \mathbb{N}}\right)=\frac{1}{2}$. Therefore $\mathcal{C} \subset \mathcal{T}_{\frac{1}{2}}$. If $\lim _{n \rightarrow \infty} n b_{n}=$ $\infty$ we have the same result for the set $\mathcal{B}$. (In the notation of paper [D3] $d\left(\left(a_{n}\right)_{n \in \mathbb{N}}\right)$ is our $1-h\left(\left(a_{n}\right)_{n \in \mathbb{N}}\right)$. Hence $\left.d\left(\left(a_{n}\right)_{n \in \mathbb{N}}\right)=\frac{1}{2} \Longleftrightarrow h\left(\left(a_{n}\right)_{n \in \mathbb{N}}\right)=\frac{1}{2}\right)$. Then corollary 3.8 with $c=\frac{1}{2}$ gives us our result.

Now one can work on this result in two different directions. Clearly, if the assumptions of Theorem 4.1 hold then $\mathcal{C} \subset \mathcal{T}_{\frac{1}{2}}$ and $\mathcal{T}_{\frac{1}{2}}$ is a closed set and hence a complete metric space with topology inherited from $\left(\{0,1\}^{\mathbb{N}}, d_{D}\right)$. Therefore we can ask what is the 'size' of the set $\mathcal{C}$ in this much smaller metric space. We will address this issue in the next theorem.

The other possible improvement of Theorem 4.1 would be lifting of the condition

$$
\varliminf_{n \rightarrow \infty} n b_{n}>0 .
$$

We address this question later. It is also interesting to compare condition (12) with condition (SLPS) from the paper [D2]. As proved there, if $\left(b_{n}\right)_{n \in \mathbb{N}}$ is nonincreasing, then (SLPS) and (12) are equivalent. Let us recall that in the paper mentioned we have showed that (SLPS) implies that the sets $\mathcal{C}$ and $\mathcal{B}$ are $\sigma$-very porous in $\left(\{0,1\}^{\mathbb{N}}, d_{B}\right)$. Now we have similar result for the D-metric with one restriction - monotonicity of the sequence $\left(b_{n}\right)_{n \in \mathbb{N}}$. It would certainly be interesting to try to lift this assumption, as well. 
Theorem 4.2. Let $\left(b_{n}\right)_{n \in \mathbb{N}}$ be a nonincreasing sequence of real numbers tending to zero satisfying the condition (12). Then the set

$$
\mathcal{C}=\left\{\left(a_{n}\right)_{n \in \mathbb{N}} ; \text { series (1) converges }\right\}
$$

is of the first Baire category in the complete metric space $\left(\mathcal{T}_{\frac{1}{2}}, d_{D}\right)$. If also $\lim _{n \rightarrow \infty} n b_{n}=\infty$ then we have the same result for the set

$$
\mathcal{B}=\left\{\left(a_{n}\right)_{n \in \mathbb{N}} ; \exists M>0 \quad \forall k \quad\left|\sum_{n=1}^{k}(-1)^{a_{n}} b_{n}\right| \leq M\right\} .
$$

This set $\mathcal{B}$ is also of type $F_{\sigma}$ in this space.

Proof. Define $\mathcal{B}_{\frac{1}{2}}=\mathcal{B} \cap \mathcal{T}_{\frac{1}{2}}$. Then we have $\mathcal{C} \subset \mathcal{B}_{\frac{1}{2}} \subset \mathcal{T}_{\frac{1}{2}}$. If we prove the statement of the theorem for the set $\mathcal{B}_{\frac{1}{2}}$, we are done. Write therefore

$$
\mathcal{B}_{\frac{1}{2}}=\bigcup_{M=1}^{\infty} \bigcap_{k=1}^{\infty}\left\{\left(a_{n}\right)_{n \in \mathbb{N}} \in \mathcal{T}_{\frac{1}{2}} ;\left|\sum_{n=1}^{k}(-1)^{a_{n}} b_{n}\right| \leq M\right\} .
$$

We define $F_{i}$ by

$$
F_{i}=\bigcap_{k=1}^{\infty}\left\{\left(a_{n}\right)_{n \in \mathbb{N}} \in \mathcal{T}_{\frac{1}{2}} ;\left|\sum_{n=1}^{k}(-1)^{a_{n}} b_{n}\right| \leq i\right\} .
$$

Clearly, $B_{\frac{1}{2}}=\cup F_{i}$. The sets $F_{i}$ are closed in the space $\left(\mathcal{T}_{\frac{1}{2}}, d_{D}\right)$ by Corollary 2.3 (Namely, the complement of $F_{i}$ could be written as a union of open sets from this corollary). Thus $B_{\frac{1}{2}}$ is a $F_{\sigma}$ set. It remains to show that each $F_{i}$ is a nowhere dense set.

Take any sequence $\left(a_{n}\right)_{n \in \mathbb{N}} \in F_{i}$. We want to show that for any $\varepsilon>0$ there is another sequence $\left(d_{n}\right)_{n \in \mathbb{N}} \in \mathcal{T}_{\frac{1}{2}}$ such that $d_{D}(a, d)<\varepsilon$ and $d=$ $\left(d_{n}\right)_{n \in \mathbb{N}} \notin F_{i}$. Here we will make use of Example 3.6 and Corollary 3.7 from [D3]. According to them for any nonincreasing sequence $\left(b_{n}\right)_{n \in \mathbb{N}}$ tending to zero whose sum is infinity there is a sequence $\left(c_{n}\right)_{n \in \mathbb{N}} \in \mathcal{T}_{\frac{1}{2}}$; i.e., $h\left(\left(c_{n}\right)_{n \in \mathbb{N}}\right)=$ $\frac{1}{2}$ for which

$$
\varlimsup_{k \rightarrow \infty} \sum_{n=1}^{k}(-1)^{c_{n}} b_{n}=+\infty \text { and } \underline{\lim }_{k \rightarrow \infty} \sum_{n=1}^{k}(-1)^{c_{n}} b_{n}=-\infty .
$$

We define the sequence $\left(d_{n}\right)_{n \in \mathbb{N}}$ using both $\left(a_{n}\right)_{n \in \mathbb{N}}$ and $\left(c_{n}\right)_{n \in \mathbb{N}}$. Let $k$ be a integer specified later and put

$$
d_{n}= \begin{cases}a_{n}, & \text { for } n \leq k \\ c_{n}, & \text { for } n>k\end{cases}
$$


The claim is that for $k$ big enough sequences $\left(a_{n}\right)_{n \in \mathbb{N}}$ and $\left(d_{n}\right)_{n \in \mathbb{N}}$ are close. To see this pick the integer $k$ such that we have

$$
\forall n \geq k:\left|\frac{a_{1}+a_{2}+\cdots+a_{n}}{n}-\frac{1}{2}\right|<\frac{\varepsilon}{4}, \text { and }\left|\frac{c_{1}+c_{2}+\cdots+c_{n}}{n}-\frac{1}{2}\right|<\frac{\varepsilon}{4}
$$

From this for any $n>k$ we have an estimate

$$
\begin{aligned}
& \left|\frac{d_{1}+d_{2}+\cdots+d_{n}-a_{1}-a_{2}-\cdots-a_{n}}{n}\right| \\
= & \left|\frac{c_{k+1}+c_{k+2}+\cdots+c_{n}-a_{k+1}-a_{k+2}-\cdots-a_{n}}{n}\right| \\
= & \left|\frac{c_{1}+c_{2}+\cdots+c_{n}}{n}-\frac{a_{1}+a_{2}+\cdots+a_{n}}{n}+\frac{a_{1}+\cdots+a_{k}}{n}-\frac{c_{1}+\cdots+c_{k}}{n}\right| \\
\leq & \left|\frac{c_{1}+c_{2}+\cdots+c_{n}}{n}-\frac{1}{2}\right|+\left|\frac{1}{2}-\frac{a_{1}+a_{2}+\cdots+a_{n}}{n}\right|+\left|\frac{a_{1}+\cdots+a_{k}}{n}-\frac{k}{2 n}\right| \\
+ & \left|\frac{k}{2 n}-\frac{c_{1}+\cdots+c_{k}}{n}\right| \leq \frac{\varepsilon}{4}+\frac{\varepsilon}{4}+\frac{k}{n} \frac{\varepsilon}{4}+\frac{k}{n} \frac{\varepsilon}{4}<\varepsilon .
\end{aligned}
$$

For $n \leq k$ there is no difference between the sequences $\left(a_{n}\right)_{n \in \mathbb{N}}$ and $\left(d_{n}\right)_{n \in \mathbb{N}}$. Together this yields $d_{D}(a, d)<\varepsilon$. The sequence $\left(d_{n}\right)_{n \in \mathbb{N}}$ clearly does not belong to any $F_{i}$ since the tails of the sequences $\left(d_{n}\right)_{n \in \mathbb{N}}$ and $\left(c_{n}\right)_{n \in \mathbb{N}}$ are same and for the sequence $\left(c_{n}\right)_{n \in \mathbb{N}}$ we have (13).

Now we want to lift condition (12). We first prove the following auxiliary result.

Proposition 4.3. Let $\left(b_{n}\right)_{n \in \mathbb{N}}$ be a nonincreasing sequence tending to zero whose sum is infinity. Let $\left(a_{n}\right)_{n \in \mathbb{N}} \in\{0,1\}^{\mathbb{N}}$ be a given sequence for which the series $\sum_{n=1}^{\infty}(-1)^{a_{n}} b_{n}$ has bounded partial sums (i.e. belongs to $\left.\mathcal{B}\right)$. Then for any $\varepsilon>0$ there is a sequence $\left(c_{n}\right)_{n \in \mathbb{N}} \in\{0,1\}^{\mathbb{N}}$ such that $d_{D}(a, c)<\varepsilon$ and $\sum_{n=1}^{\infty}(-1)^{c_{n}} b_{n}$ diverges either to $+\infty$ or $-\infty$.

Proof. The sequence $\left(c_{n}\right)_{n \in \mathbb{N}}$ will be defined by a slight modification of the given sequence $\left(a_{n}\right)_{n \in \mathbb{N}}$. The idea behind our construction will be similar to the one used the proof of Theorem 3.5. Our first claim is that

$$
\sum_{i=1}^{\infty} 2^{i} b_{2^{i}}=\infty
$$


To see this consider

$$
\begin{aligned}
\sum_{i=0}^{\infty} 2^{i} b_{2^{i}} & =b_{1}+\left(b_{2}+b_{2}\right)+(\underbrace{b_{4}+\cdots+b_{4}}_{4 \text { times }})+(\underbrace{b_{8}+\cdots+b_{8}}_{8 \text { times }})+\ldots \\
& \geq b_{1}+\left(b_{2}+b_{3}\right)+\left(b_{4}+b_{5}+\cdots+b_{7}\right)+\left(b_{8}+b_{9}+\cdots+b_{15}\right)+\ldots \\
& =\sum_{n=1}^{\infty} b_{n}=\infty .
\end{aligned}
$$

Now take any given $\varepsilon>0$ and find a positive integer $k$ such that $\frac{1}{2^{k}}<$ $\frac{\varepsilon}{2}$. For each $j \geq k+1$ there are at least $2^{j-k}$ zeros or at least $2^{j-k}$ ones among the numbers $a_{2^{j}+1}, a_{2^{j}+2}, a_{2^{j}+3}, \ldots, a_{2^{j}+2^{j}}$. Now we sort out the set of the integers $j \geq k+1$ into two increasing sequences of integers $x_{1}<x_{2}<$ $x_{3}<\ldots$, and $y_{1}<y_{2}<y_{3}<\ldots$, such that an integer $j \geq k+1$ is in the sequence $\left(x_{n}\right)_{n \in I_{1}}$ if there are at least $2^{j-k}$ zeros among the numbers $a_{2^{j}+1}, a_{2^{j}+2}, a_{2^{j}+3}, \ldots, a_{2^{j}+2^{j}}$, and in the sequence $\left(y_{n}\right)_{n \in I_{2}}$ otherwise. Of course it is possible that one of these two sequences is finite. But by (14)

$$
\infty=\sum_{j \geq k+1}^{\infty} 2^{j} b_{2^{j+1}}=\sum_{n \in I_{1}} 2^{x_{n}} b_{2^{x_{n}+1}}+\sum_{n \in I_{2}} 2^{y_{n}} b_{2^{y_{n}+1}} .
$$

This means that at least one of the sums on the right hand side is infinite. Without loss of generality assume that this is true for the first one. It follows that the sequence $\left(x_{n}\right)_{n \in I_{1}}$ is infinite and the set of indices $I_{1}$ is just $\mathbb{N}$. Now we can define our sequence $\left(c_{n}\right)_{n \in \mathbb{N}}$. First we put

$$
c_{n}= \begin{cases}a_{n}, & \text { for } n=1,2, \ldots, 2^{k+1}, \\ a_{n}, & \text { if } 2^{y_{j}}<n \leq 2^{y_{j}+1} \text { for some } j \in I_{2} .\end{cases}
$$

So it remains to define $\left(c_{n}\right)_{n \in \mathbb{N}}$ for $2^{x_{j}}<n \leq 2^{x_{j}+1}, j=1,2,3, \ldots$ From the definition of the number $x_{j}$ there are at least $2^{x_{j}-k}$ zeros among the numbers $a_{2^{x_{j}}+1}, a_{2^{x_{j}}+2}, a_{2^{x_{j}}+3}, \ldots, a_{2^{x_{j}+1}}$. Among those zeros pick exactly the first $2^{x_{j}-k}$ of them and change them to ones. For the remaining numbers $n$ just take $c_{n}=a_{n}$. Now the sequence $\left(c_{n}\right)_{n \in \mathbb{N}}$ is defined everywhere. It remains to be seen that such a sequence gives rise to a divergent series (to $-\infty)$ and that its distance from $\left(a_{n}\right)_{n \in \mathbb{N}}$ in the D-metric is less than $\varepsilon$.

First let us look at the convergence of such series. We know that the original sequence $\left(a_{n}\right)_{n \in \mathbb{N}}$ gave us a series (1) with bounded partial sums which means that there exist $M>0$ such that $\left|s_{n}\right|=\left|\sum_{k=1}^{n}(-1)^{a_{k}} b_{k}\right| \leq M$, for $n=1,2, \ldots$. Therefore we have $\sum_{n=1}^{\infty}(-1)^{c_{n}} b_{n} \leq M-\sum_{n \in \mathbb{N} ; c_{n} \neq a_{n}} 2 b_{n}$. 
If we prove that $\sum_{n \in \mathbb{N} ; c_{n} \neq a_{n}} b_{n}=\infty$, then we are done, since the divergence of the series $\sum_{n=1}^{\infty}(-1)^{c_{n}} b_{n}$ to $-\infty$ follows. Among the numbers $n$ between $2^{x_{j}}<n \leq 2^{x_{j}+1}$ there are exactly $2^{x_{j}-k}$ of them for which $c_{n} \neq a_{n}$. This and the monotonicity of $\left(b_{n}\right)_{n \in \mathbb{N}}$ gives us

$$
\sum_{n \in \mathbb{N} ; c_{n} \neq a_{n}} b_{n} \geq \sum_{j=1}^{\infty} 2^{x_{j}-k} b_{2^{x_{j}+1}} \geq \frac{1}{2^{k}} \sum_{j=1}^{\infty} 2^{x_{j}} b_{2^{x_{j}+1}}=\infty .
$$

The last equality comes from the way the sequence $\left(x_{j}\right)_{j \in \mathbb{N}}$ was picked.

We show now, that the series $\left(c_{n}\right)_{n \in \mathbb{N}}$ is not 'far away' from $\left(a_{n}\right)_{n \in \mathbb{N}}$ in the D-metric. To see this let us evaluate

$$
\frac{c_{1}+c_{2}+c_{3}+\cdots+c_{n}-a_{1}-a_{2}-a_{3}-\cdots-a_{n}}{n} .
$$

We can see that the critical $n$ where this number has to be estimated are those $n$ between $2^{x_{j}}<n \leq 2^{x_{j}+1}$ for some $j=1,2,3, \ldots$, because only there could $a_{n} \neq c_{n}$. For such $n$ we get

$\frac{c_{1}+\cdots+c_{n}-a_{1}-\cdots-a_{n}}{n}=\frac{\sum_{i=1, c_{i} \neq a_{i}}^{n} 1}{n} \leq \frac{\sum_{i=1}^{j} 2^{x_{i}-k}}{2^{x_{j}}}<\frac{1}{2^{k}} \frac{2^{x_{j}+1}}{2^{x_{j}}} \leq \frac{2}{2^{k}}<\varepsilon$.

Hence $d_{D}(a, c)<\varepsilon$ follows.

This result leads to the final assertion.

Theorem 4.4. Let $\left(b_{n}\right)_{n \in \mathbb{N}}$ be a nonincreasing sequence tending to zero whose sum is infinity. Then the sets

$$
\mathcal{C}=\left\{\left(a_{n}\right)_{n \in \mathbb{N}} \in\{0,1\}^{\mathbb{N}} ; \text { series (1) converges }\right\},
$$

and

$$
\mathcal{B}=\left\{\left(a_{n}\right)_{n \in \mathbb{N}} \in\{0,1\}^{\mathbb{N}} ; \exists M>0 \quad \forall k\left|\sum_{n=1}^{k}(-1)^{a_{n}} b_{n}\right| \leq M\right\},
$$

are of the first Baire category the complete metric space $\left(\{0,1\}^{\mathbb{N}}, d_{D}\right)$. The set $\mathcal{B}$ is also of type $F_{\sigma}$ in this space.

Proof. Again it is sufficient to prove our theorem only for the set $\mathcal{B}$ since $\mathcal{C} \subset \mathcal{B}$. Write the set $\mathcal{B}$ as in the proof of Theorem 4.2

$$
\mathcal{B}=\bigcup_{M=1}^{\infty} \bigcap_{k=1}^{\infty}\left\{\left(a_{n}\right)_{n \in \mathbb{N}} ;\left|\sum_{n=1}^{k}(-1)^{a_{n}} b_{n}\right| \leq M\right\}
$$


We define $F_{i}$ by $F_{i}=\bigcap_{k=1}^{\infty}\left\{\left(a_{n}\right)_{n \in \mathbb{N}} ;\left|\sum_{n=1}^{k}(-1)^{a_{n}} b_{n}\right| \leq i\right\}$. Then clearly, $B=\cup F_{i}$. The sets $F_{i}$ are again closed. It remains to be seen that they are nowhere dense.

Take any sequence $\left(a_{n}\right)_{n \in \mathbb{N}} \in F_{i}$. We want to show that for any $\varepsilon>0$ there is another sequence $\left(c_{n}\right)_{n \in \mathbb{N}}$ such that $d(a, c)<\varepsilon$ and $c=\left(c_{n}\right)_{n \in \mathbb{N}} \notin F_{i}$. But the existence of such sequence is guaranteed by Proposition 4.3 and therefore the set $F_{i}$ is indeed nowhere dense. Hence our theorem is proved.

As examples of series where previous theorems are applicable could serve

$$
\sum_{n=1}^{\infty}(-1)^{a_{n}} \frac{1}{n} \text { or } \sum_{n=2}^{\infty}(-1)^{a_{n}} \frac{1}{\ln n} .
$$

\section{References}

[B] P. Bundschuh, Konvergenz unendlicher Reihen und Gleeichverteillung mod 1, Arch. Math. 29 (1977), 518-523.

[C] E. Cesàro, Sur une distribution de signes, Rend. Acc. Lincei 41, (1888) 133.

[D1] M. Dindoš, On series with alternating signs in Euclidean metric, Real Anal. Exch., 25 (1999-2000), 599-616.

[D2] M. Dindoš, On a typical series with alternating signs, Real Anal. Exch., 25 (1999-2000), 617-628.

[D3] M. Dindoš, On sign distribution in relatively convergent series, Acta. Math. Hung., to appear.

[H] G. H. Hardy, Note on a theorem of Cesàro, Messenger Math 41 (1912), $17-22$.

[LŠ] V. László and T. Šalát, The structure of some sequence spaces, and uniform distribution (mod 1), Period. Math. Hung., 10 (1979), 89-98.

[R] J. F. Randolph, Basic real and abstract analysis, Academic press Inc., New York and London (1968).

[ک̌] T. Šalát, On subseries of divergent series, Matematický časopis, 18 (1968), 312-338.

[Z] V. Zanelli, Sign distribution and absolutely divergent series, Periodica Mathematica Hungarica, 28(1) (1994), 1-17. 